\title{
Impact of Management Factors on Seminal Attributes and Frozen Semen Doses Production in Holstein Friesian Crossbred Dairy Bulls
}

Dilip Kumar Mandal ( $\nabla$ dkmandal1998@gmail.com )

ICAR-National Dairy Research Institute https://orcid.org/0000-0001-5292-1109

Mahesh Kumar

ICAR-Central Institute for Research on Cattle, Meerut

Shrikant Tyagi

ICAR-Central Institute for Research on Cattle, Meerut

\section{Research Article}

Keywords: Semen quality, sperm morphology, season and age, Holstein Friesian X Sahiwal crossbred dairy bull, frozen semen doses

Posted Date: August 4th, 2021

DOI: https://doi.org/10.21203/rs.3.rs-747077/v1

License: (c) (1) This work is licensed under a Creative Commons Attribution 4.0 International License. Read Full License 


\section{Abstract}

This study reports on important management factors that have impacts on semen characteristics, sperm production ability and cryo-preservability in dairy bulls. Except volume, bull types (FSD i.e. frozen semen dose producer vs Non-FSD bulls) differentiated most of the semen traits $(P<0.01)$. Age showed significant impacts on seminal attributes both in FSD and Non-FSD bulls. In FSD bulls semen traits increased significantly even up to 5 years age, in contrast, only 2 years in Non-FSD bulls. Overall semen quality index improved almost linearly with age advancement in FSD bulls $(P<0.01)$ but not in NonFSD bulls, which showed very irrational trends. Seasons significantly $(P<0.01)$ influenced ejaculate characteristics. Overall semen quality, total sperm output and FSD production was the highest during summer and lowest in rainy seasons. Within a bull type (FSD/non-FSD) influence of age and season, and within age group impact of seasons was significant in most of the semen attributes. It was concluded that dependent on season, age of bull significantly influenced qualitative and quantitative attributes of semen; improvement being substantial in FSD bulls, but negligible in non-FSD bulls beyond 2 years age. Seasons of collection significantly influenced semen quality, sperm productivity and FSD production of bulls being highest in summer, followed by winter and least in rainy seasons. The study recommended that for effective FSD production, poor semen producer bulls should be culled as early as 2 years of age and during rainy season stress amelioration measures might mitigate FSD production losses from crossbred bulls.

\section{Introduction}

The purpose of introduction of temperate dairy cattle breeds to tropical countries was to improve milk production through crossbreeding. Among temperate dairy breeds Holstein Friesian (Holstein / Friesian) has been extensively used as improver breed in India, Pakistan, Iraq among Asian countries, Egypt, Sudan, Ethiopia, Kenya, Tanzania, Nigeria among African countries, Brazil, Bolivia, Columbia, Jamaica, Cuba, El-Salvador and others in Latin American Countries. Worldwide a sizable number of crossbred dairy cow populations have Holstein Friesian (HF) inheritance owing to either systematic or non-systematic cattle breeding programmes adopted by different countries. A series of crossbreeding experiments in cattle had been carried out in India and other countries for genetic improvement, which resulted into enhancement of milk production traits, but invited problems of more diseases incidence, reproductive problems etc. in cows. Major problems encountered with semen production from crossbred dairy bulls are very high disposal rate due to unsatisfactory breeding soundness, sub fertility, unsatisfactory semen quality and insufficient capacity to sustain cryo-preservation i.e. semen nonfreezability (Chacko, 2005; Mandal et al. 2012; Singh et al. 2013). Therefore, to have a better insight on problems of quality semen production from crossbred bulls, important factors affecting reproductive performance in bulls need detailed investigation in tropical environment, because crossbred cows are the major producers of cow-milk in India and other developing countries.

Breed, age, climatic conditions of the region, micro-climatic circumstances like feeding and general management of the farm, very short term events like sexual activity, preparation, bull handling during semen collection etc. affect semen production (Everett and Bean, 1982; Mandal 1998; Kastelic, 2013; Argiris et al. 2018). Seasonal influence on sperm characteristics is remarkably wide being highly significant to no prominent impact (Everett and Bean, 1982; Mandal 1998; Brito et al. 2002a; Mandal et al. 2003; Chauhan et al. 2010). For example, frozen semen doses (FSD) prepared in stressful season had apparently normal quality, however, showed reduced embryo development in vitro (Luceño et al. 2020). In South East Brazilian climatic conditions semen collection from $B$. taurus bulls for FSD production may not be profitable during summer months due to quality deterioration (Koivisto et al. 2009).

Studies on seasonality on semen quality of dairy bulls had created renewed interest because natural heat stress of summer even from temperate countries reported to reduce semen quality (Sabés-Alsina et al. 2017; Luceño et al. 2020). Season induced negative effects were deterioration of sperm morphology, alteration of lipid composition at cellular and semen plasma level (Argov-Argaman et al. 2013), reduced mRNA expression of very low density lipid receptors on sperm membrane (Argov et al. 2007), changes in sperm kinematics, membrane integrity, mitochondrial membrane potential, 
acrosome structure (Orgal et al. 2012; Argov-Argaman et al. 2013; Sabés-Alsina et al. 2017, 2019) and alteration in electrolytes in seminal plasma (Orgal et al. 2012). Some of the seasonal changes are not visible in fresh ejaculates but evident in post-thaw semen quality like reduced sperm kinematic characteristics and probably similar to that of subtle sperm defects not visible in normal sperm morphology assessment but their impacts are even non-compensable by enhanced number of spermatozoa in artificial insemination (Al) to attain desired fertility rates (Barth and Oko, 1989). These negative effects could be mitigated to some extent by strategic bull husbandry (Morrell et al. 2020), which gave impetus for meticulously study on the important management factors that affect semen production, more so when reproductive wastage, semen freezability and FSD production from crossbred bulls are serious problems under tropical environment conditions (Chacko, 2005; Singh et al. 2013).

Frozen semen dose (FSD) production centers are quite interested in production of maximum numbers of semen doses from elite bulls for economic reasons. In a given environment, productivity and adaptive efficiency of bulls depends largely on reproductive capacity; and poor reproductive performances become the cause of culling, loss of production, poor fertility and substantial economic losses. Thus, a clear understanding of reproductive process and factors that affect reproductive ability of bulls are very useful for production of quality FSDs and success of artificial insemination (Al). Besides, studies on management factors affecting seminal characteristics will help policy makers in planning and making accurate decisions on bull management, number of bulls to be reared at FSD production stations, harvest of required number of FSD, supply of desired number of FSD from chosen bulls in Al programme and profitability of bull station. Therefore, the aim of this study was to identify various management factors viz. season, age, ejaculate collection frequency etc. that could influence the semen characteristics, sperm production capacity and FSD production of crossbred dairy bulls.

\section{Material And Methods \\ 2.1. Location of farm}

Present study was conducted at Bull Rearing Unit, Central Institute for Research on Cattle, Uttar Pradesh, Meerut, India.

\subsection{Year and season}

Seminal ejaculates $(n=14328)$ used in this study were (year 2000-2008) of 269 Holstein Friesian X Sahiwal crossbred dairy bulls. According to temperature and rainfall patterns of the farm location, the year was divided into 3 seasons i.e. summer (March-June), rainy (July-October) and winter (November-February).

\subsection{Breed and bull type}

Animals under the study ( $\mathrm{N}=269)$ were Holstein Friesian (HF) X Sahiwal crossbred dairy bulls having breed combination of $\simeq 5 / 8 \mathrm{HF}$ (Bos taurus) and 3/8 Sahiwal (Bos indicus) that stabilized over several generations. They are popularly named as 'Frieswal' dairy bulls. Bulls were categorized into 2 groups depending upon their semen freezability and frozen semen doses (FSD) production. Bulls whose seminal ejaculates yielded desirable post-thaw sperm motility ( $\geq 40 \%)$ and produced FSDs were termed as FSD bulls $(\mathrm{N}=133)$ and those had unacceptable post-thaw sperm motility $(<40 \%)$, hence no FSDs were produced from any of the ejaculates during their herd-life were termed as Non-FSD bulls $(\mathrm{N}=136)$.

\subsection{Age}

The age of bulls ranged from 15 to 93 months in this study. Age was divided into 5 brackets: $\leq 24$ months, 25-36 m, 37$48 \mathrm{~m}, 49-60 \mathrm{~m}$ and $>60 \mathrm{~m}$ keeping 1-year intervals.

\subsection{General management of bulls}

Housing of bulls was in individual pens covered with asbestos roof and open loafing area for free movement within their enclosure. Bulls were given periodic exercise for one hour once a week in a bull exerciser. Bulls were given anthelmentics twice a year and vaccinated against FMD, HS\&BQ as routine. Seasonal green fodder (ad libitum), wheat bhusa (finely 
chaffed wheat straw) constituted the roughage portion of the diet and amount of concentrate adjusted to body weight of individual bull as per routine schedule of the farm. Bulls were thoroughly washed with water, particularly ventral part and prepuce 1-2 hour prior to semen collection. In case of second ejaculation, a gap of 30 minutes was given from first ejaculation and till then bulls were kept tied at holding area of collection centre.

\subsection{Collection of semen and quality evaluation}

At 15-18 months of age bulls' semen collection was initiated. Bulls refused to mount, having poor libido, very inconsistent semen donation or stayed less than 6 months in collection programme were excluded in this study.

In young bulls ( $\leq 30 \mathrm{~m}$ age) semen collection was done once a week and in mature bulls $(>30 \mathrm{~m})$ twice a week. In few mature bulls 2 successive ejaculates in a day were collected at a gap of 30 minutes. Semen was collected at 7:00-9:00 a.m. by using artificial vagina (internal temperature $42-45^{\circ} \mathrm{C}$ ). Ejaculates were evaluate for volume ( $\mathrm{ml}$ ), colour (5 categories: Creamy, Light creamy/Cloudy, Milky, Yellow and Watery) and consistency (3 Categories: Thick, Medium and Thin). Sperm concentration (million /ml) was determined using a sperm photometer (Accucell, IMV). Sperm progressive motility (\%) evaluation was carried out by using a DIC microscope. Ejaculates ( $\geq 60 \%$ sperm progressive motility) were processed for cryopreservation as per standard process (Hafez, 1993). Post-thaw evaluation of FSDs done 24 hours later and frozen semen doses $\geq 40 \%$ post thaw sperm motility were accepted for use in Al, otherwise doses were discarded.

\subsection{Semen Quality Index (SQI; 0-100 scale)}

In most of the FSD production stations initial semen quality is evaluated based on physical attributes (colour, consistency) and objective assessment viz. volume, sperm motility \% and sperm concentration. We have developed semen quality index (SQI) to determine overall assessment of ejaculates implying suitable scores to VOL, MOT and SC based on our experience and rational described as under.

\begin{tabular}{|llllll|}
\hline VOL & Score & SC (million/ml) & Score & MOT (\%) & Score \\
\hline$\geq 5 \mathrm{ml}$ & 20 & $\geq 1200$ & 30 & $\geq 70$ & 50 \\
\hline $5 \mathrm{ml}$ & 10 & $\geq 900$ to $<1200$ & 20 & $\geq 60$ to $<70$ & 40 \\
& & $\geq 600$ to $<900$ & 10 & $\geq 60$ to $<50$ & 30 \\
& & 0 & $\geq 50$ to $<40$ & 20 \\
& & & & $\geq 30$ to $<40$ & 10 \\
& & & & $\geq 20$ to $<30$ & 0 \\
& & & $<20$ & -10 \\
\hline
\end{tabular}

For example, (i) an ejaculate of $5 \mathrm{ml}$, SC of $1200 \mathrm{million} / \mathrm{ml}$ and MOT 70\%, obtained SQI $=20+30+50=100$ (ii) an ejaculate of $4 \mathrm{ml}, \mathrm{SC} 590 \mathrm{million} / \mathrm{ml}$ and MOT below $20 \%$, obtained SQI $=10+0+(-10)=0$.

Bulls initial sperm motility below $20 \%$ have questionable fertility even at natural mating, practically encompass no breeding use and thus given negative score of minus 10. For breeding soundness evaluation minimum standard of sperm motility was set to $\geq 30 \%$ (Kastelic 2013). Hence, for SQI estimation MOT < 30 was given score zero and MOT $<20$, we have assigned - 10 score to make poor quality ejaculates SQI value least possible or zero.

\subsection{Statistical analysis}

Semen quality parameters viz. volume (VOL-in ml), sperm concentration (SC-in million), progressive motile sperm \% (MOT), semen quality index (SQI), total sperm output / ejaculate (TSO; in millions), total motile sperm /ejaculate (TSM; in millions), post-thaw motility \% (MOT-PT) and post thaw total motile sperm count / ejaculate (TSM-PT; in millions) were analyzed by 
using GLM univariate analysis of variance (SPSS, 2007). Percentage values of parameters viz. MOT, MOT-PT were transformed using arcsine transformation techniques (Snedecor and Cochran, 1994). We used following model for statistical analysis.

$Y_{i j k l m}=\mu+B_{i}+A_{j}+S_{k}+F_{l}+(B A)_{i j}+(B S)_{i k}+(A S)_{j k}+e_{i j k l m}$

Where,

$\mu=$ Overall mean,

$B_{i}=$ Effect of $i^{\text {th }}$ bull type $(i=1,2 ;$ FSD bull or Non-FSD bulls),

$A_{j}=$ Effect of $j^{\text {th }}$ age group $(j=1, \ldots . ., 5)$

$S_{k}=$ Effect of $k^{\text {th }}$ season $(k=1, . ., 3)$

$F_{I}=$ Effect of $I^{\text {th }}$ ejaculation on same day $(I=1,2$; first or second),

$(B A)_{i j}=$ Interaction between $\mathrm{i}^{\text {th }}$ bull type and $\mathrm{j}^{\text {th }}$ age group,

$(B S)_{i k}=$ Interaction between $\mathrm{i}^{\text {th }}$ bull type and $\mathrm{k}^{\text {th }}$ season,

$(A S)_{j k}=$ Interaction between $j^{\text {th }}$ age group and $k^{\text {th }}$ season,

$\mathrm{e}_{\mathrm{ijklm}}=$ Random error part of $\mathrm{Y}_{\mathrm{ijklm}}$

The same above model was used for analysis of management factors affecting FSD production, excluding the bull types. Levels of significance was set at $P<0.05$ and $P<0.01$. Scheffe's test was used for multiple comparison of mean significant difference. Pearson's correlation coefficient $(r)$ was estimated for association studies between age and seminal parameters. Chi-Square $\left(\chi^{2}\right)$ test of independence was used for analysis of frequency data.

\section{Results}

The overall (mean $\pm \mathrm{SE}$ ) semen quality viz. ejaculate volume (VOL), sperm concentration /ml (SC), progressive motile sperm \% (MOT), semen quality index (SQl; 0-100 scale), TSO / ejaculate, TSM / per ejaculate, post-thaw motility \% (MOT-PT) and post thaw total motile sperm count per ejaculate (TSM-PT) were $4.61 \pm 0.02 \mathrm{ml}, 1081.75 \pm 4.84$ million, $44.66 \pm 0.17,57.29$ $\pm 0.23,5146.27 \pm 31.88$ million, $2538.71 \pm 19.39$ million, $37.82 \pm 0.25$ and $2312.89 \pm 27.18$ million, respectively. The overall (mean \pm SE) number of frozen semen doses production was $244.96 \pm 2.89$ per ejaculate. The effects of bull type, age, season, ejaculate frequency and their interactions on seminal attributes are presented in Table 1. Ejaculates' colour showed significant variations $\left(X_{d f, 16}^{2}=466.00, P<0.01\right)$ among age groups. Overall basis, $65.5 \%$ ejaculates were creamy, $11.3 \%$ light creamy, $1.10 \%$ milky, $1.30 \%$ yellow and $20.80 \%$ watery. Irrespective of age group creamy was the predominant colour in ejaculates. With advancement of age, frequency of creamy colour increased being $55.7 \%$ at 2 nd year, $60.8 \%$ at $3 \mathrm{rd}, 67.3 \%$ at 4 th, $72.7 \%$ at 5 th and $91.8 \%$ at $>5$ years of age. Seasonal variations $\left(\chi^{2} d f, 8=90.43, P<0.01\right)$ in colour of ejaculates were also evident in this study. However, frequencies of creamy coloured ejaculates did not differ among summer (65.8\%), rainy $(65.7 \%)$ and winter $(65.3 \%)$ seasons. 
Table 1

Significance levels ofdifferent factors influencing semen traits of crossbred dairy bulls

\begin{tabular}{|c|c|c|c|c|c|c|c|c|}
\hline Particulars & $\begin{array}{l}\text { VOL } \\
(\mathrm{ml})\end{array}$ & $\begin{array}{l}\text { SC } \\
\text { (million } \\
\text { /ml) }\end{array}$ & $\begin{array}{l}\text { Progressive sperm } \\
\text { Motility \% (MOT) }\end{array}$ & SQI & $\begin{array}{l}\text { TSO/ } \\
\text { ejaculate } \\
\text { (million) }\end{array}$ & $\begin{array}{l}\text { TSM/ } \\
\text { ejaculate } \\
\text { (million) }\end{array}$ & $\begin{array}{l}\text { MOT- } \\
\text { PT } \\
(\%)\end{array}$ & $\begin{array}{l}\text { TSM-PT/ } \\
\text { ejaculate } \\
\text { (million) }\end{array}$ \\
\hline $\begin{array}{l}\text { Effect of Bull } \\
\text { type }\end{array}$ & NS & $\star \star$ & $\star \star$ & $\star *$ & $\star \star$ & $\star *$ & $\star *$ & $\star \star$ \\
\hline Effect of Age & $\star \star$ & $\star *$ & $\star \star$ & $\star \star$ & $\star \star$ & $\star \star$ & $\star *$ & $\star \star$ \\
\hline $\begin{array}{l}\text { Effect of } \\
\text { Season }\end{array}$ & NS & ** & NS & ** & $\star \star$ & $\star \star$ & NS & * \\
\hline $\begin{array}{l}\text { Effect of } \\
\text { ejaculate } \\
\text { number }\end{array}$ & ** & $\star \star$ & $\star \star$ & NS & $\star \star$ & $\star \star$ & ** & $\star \star$ \\
\hline $\begin{array}{l}\text { Bull type X Age } \\
\text { interaction }\end{array}$ & ** & $\star *$ & * & $\star \star$ & $\star \star$ & $\star \star$ & NS & NS \\
\hline $\begin{array}{l}\text { Bull type X } \\
\text { Season } \\
\text { interaction }\end{array}$ & ** & NS & $\star *$ & * & $\star *$ & $\star \star$ & NS & NS \\
\hline $\begin{array}{l}\text { Age X Season } \\
\text { interaction }\end{array}$ & ** & $\star \star$ & ** & $\star \star$ & ** & $\star \star$ & NS & ** \\
\hline \multicolumn{9}{|c|}{$\begin{array}{l}\text { * Significant }(P<0.01) \text {, * significant }(p<0.05) \text {, NS: non-significant }(p>0.05) \text {, VOL; Volume, SC: Sperm concentration, } \\
\text { MOT: Progressive sperm motility \%, SQI: semen quality index }(0-100 \text { scale), TSO: total sperm output per ejaculate, TSM } \\
\text { total motile sperm per ejaculate, MOT-PT: post-thaw motility \%, TSM-PT: post thaw total motile sperm count per } \\
\text { ejaculate }\end{array}$} \\
\hline
\end{tabular}

The consistency of ejaculates showed significant variations $\left(\chi^{2} d f, 12=6274.00, P<0.01\right)$ among age groups. Overall basis, $42.8 \%$ ejaculates were thick, $35.6 \%$ medium, $20.4 \%$ thin and $1.2 \%$ watery. At 2 years of age group, $34.1 \%$ ejaculates were thin, $34.3 \%$ medium and $29.4 \%$ thick in consistency. At the age $>5$ years group, the values were $6.5,30.0$ and $63.6 \%$, respectively indicating increase from thin to thick consistency over years. The distribution of consistency of ejaculates varied significantly $\left(X_{d f, 6}^{2}=144.00, P<0.01\right)$ over seasons also. Thick consistency was the highest in winter $(47.4 \%)$ followed by summer (42.6\%) and lowest in rainy (36.5\%). Medium and thin consistency ejaculates were highest in rainy season as compared to summer or winter. Watery consistency (1-1.5\%) ejaculates showed almost similar distributions over seasons.

\subsection{Effect of bull types}

Differences existed between FSD and Non-FSD bulls on all seminal attributes $(P<0.05)$ except VOL $(P>0.05)$. In FSD bulls, $\mathrm{SC} / \mathrm{ml}$ and TSO/ejaculate were 40.70 and $48.69 \%$ higher as compared to that of Non-FSD bulls (Table 2). Similarly, MOT, SQI and MOT-PT in FSD bulls were double $(P<0.01)$ than that of Non-FSD bulls. Seminal ejaculates of FSD bulls sustained better the stress of freezing (cryo-preservation) and thawing, and yielded 2.45 folds higher $(P<0.01)$ post-thaw motile sperm per ejaculate as compared to Non-FSD bulls (Table 2). 
Table 2

Bull type and ejaculation number's impact on semen traits

\begin{tabular}{|c|c|c|c|c|}
\hline \multirow[b]{2}{*}{ Particulars } & \multicolumn{2}{|c|}{ Effect of type of bulls } & \multicolumn{2}{|c|}{ Effect of ejaculate frequency } \\
\hline & $\begin{array}{l}\text { FSD bulls }(n= \\
10825)\end{array}$ & $\begin{array}{l}\text { Non-FSD bulls }(\mathrm{n} \\
=3503)\end{array}$ & $\begin{array}{l}\text { Ejaculate-1 }(n= \\
\text { 12625) }\end{array}$ & $\begin{array}{l}\text { Ejaculate-2 }(n= \\
\text { 1703) }\end{array}$ \\
\hline Ejaculate volume (ml) & $4.68^{a} \pm 0.02$ & $4.39^{a} \pm 0.03$ & $4.61^{a} \pm 0.02$ & $4.57^{\mathrm{b}} \pm 0.04$ \\
\hline Concentration (million /ml) & $\begin{array}{l}1164.08^{\mathrm{a}} \pm \\
5.43\end{array}$ & $827.32^{b} \pm 9.29$ & $1100.58^{a} \pm 5.23$ & $942.13^{b} \pm 11.89$ \\
\hline Progressive Motility (\%) & $51.02^{a} \pm 0.17$ & $25.02^{b} \pm 0.32$ & $42.90^{a} \pm 0.19$ & $57.68^{b} \pm 0.35$ \\
\hline SQI (0-100) & $65.02^{\mathrm{a}} \pm 0.22$ & $33.40^{\mathrm{b}} \pm 0.42$ & $56.02^{\mathrm{a}} \pm 0.25$ & $66.72^{a} \pm 0.49$ \\
\hline $\begin{array}{l}\text { Total Sperm Output per ejaculate } \\
\text { (million) }\end{array}$ & $\begin{array}{l}5594.12^{\mathrm{a}} \pm \\
37.09\end{array}$ & $3762.32^{b} \pm 56.05$ & $\begin{array}{l}5247.97^{\mathrm{a}} \pm \\
34.69\end{array}$ & $\begin{array}{l}4392.32^{\mathrm{b}} \pm \\
73.51\end{array}$ \\
\hline $\begin{array}{l}\text { Total progressive motile sperm per } \\
\text { ejaculate (million) }\end{array}$ & $\begin{array}{l}2992.32^{\mathrm{a}} \pm \\
22.7\end{array}$ & $1136.96^{\mathrm{b}} \pm 25.08$ & $\begin{array}{l}2529.48^{\mathrm{a}} \pm \\
21.06\end{array}$ & $\begin{array}{l}2607.17^{\mathrm{b}} \pm \\
47.23^{-}\end{array}$ \\
\hline Post thaw sperm motility (\%) & $\begin{array}{l}38.30^{a} \pm 0.25 \\
(n=4257)\end{array}$ & $18.57^{b} \pm 0.96$ & $\begin{array}{l}36.08^{a} \pm 0.28 \\
(n=3495)\end{array}$ & $\begin{array}{l}44.85^{\mathrm{b}} \pm 0.48 \\
(\mathrm{n}=867)\end{array}$ \\
\hline $\begin{array}{l}\text { Total post-thaw motile sperm per } \\
\text { ejaculate (million) }\end{array}$ & $\begin{array}{l}2346.35^{a} \pm \\
27.55 \\
(n=4257)\end{array}$ & $\begin{array}{l}956.40^{b} \pm 94.9 \\
(n=105)\end{array}$ & $\begin{array}{l}2340.44^{\mathrm{a}} \pm \\
31.39 \\
(n=3495)\end{array}$ & $\begin{array}{l}2201.82^{\mathrm{b}} \pm \\
51.63 \\
(\mathrm{n}=867)\end{array}$ \\
\hline $\begin{array}{l}\text { Frozen semen doses production (FSD)/ } \\
\text { ejaculate }\end{array}$ & $244.96 \pm 2.89$ & - & $262.84^{\mathrm{a}} \pm 3.55$ & $198.09^{\mathrm{b}} \pm 4.27$ \\
\hline
\end{tabular}

\subsection{Effect of season}

Table 3 illustrate that seasons significantly $(\mathrm{P}<0.01)$ influence SC, TSO, TSM and TSM-PT but not the VOL, MOT and MOTPT. The semen quality index (SQI) was the highest in summer and lowest in rainy seasons. SC was the highest in summer, followed by winter and lowest in rainy season $(P<0.01)$. As compared to rainy season, total sperm output / ejaculate was $19.88 \%$ and $15.23 \%$ higher $(P<0.01)$ in summer and winter seasons, respectively. Seasons significantly affected the FSD production per ejaculate $\left(F_{2,2385}=15.85, P<0.01\right)$. It was lowest in rainy $(214.33 \pm 5.45)$ season. During winter and summer seasons FSD production was $16-20 \%$ higher as compared to rainy seasons (Table 3). Significant interaction of bull type $x$ season (Table 4) on VOL, SC, MOT, TSO and TSM indicated that effect of season was not similar on FSD and Non-FSD bulls. 
Table 3

Impact of season and age of bulls on semen attributes

\section{Seasons}

\begin{tabular}{|c|c|c|c|}
\hline Particulars & $\begin{array}{l}\text { Summer }(n= \\
\text { 4846) }\end{array}$ & $\begin{array}{l}\text { Rainy }(n= \\
\text { 3996) }\end{array}$ & $\begin{array}{l}\text { Winter }(n= \\
5486)\end{array}$ \\
\hline Ejaculate volume (ml) & $4.69^{a} \pm 0.03$ & $4.46^{\mathrm{a}} \pm 0.03$ & $4.65^{a} \pm 0.03$ \\
\hline Concentration (million /ml) & $\begin{array}{l}1121.50^{\mathrm{a}} \pm \\
8.74\end{array}$ & $\begin{array}{l}1013.28^{\mathrm{b}} \pm \\
8.74\end{array}$ & $\begin{array}{l}1096.50^{\mathrm{a}} \pm \\
7.67\end{array}$ \\
\hline Progressive Motility (\%) & $44.77^{a} \pm 0.31$ & $\begin{array}{l}43.61^{\mathrm{a}} \pm \\
0.33\end{array}$ & $\begin{array}{l}45.33^{\mathrm{a}} \pm \\
0.28\end{array}$ \\
\hline SQI (0-100) & $58.09^{a} \pm 0.40$ & $\begin{array}{l}54.80^{\mathrm{b}} \pm \\
0.42\end{array}$ & $\begin{array}{l}58.41^{\mathrm{a}} \pm \\
0.36\end{array}$ \\
\hline $\begin{array}{l}\text { Total Sperm Output per ejaculate } \\
\text { (million) }\end{array}$ & $\begin{array}{l}5481.02^{\mathrm{a}} \pm \\
59.55\end{array}$ & $\begin{array}{l}4572.22^{\mathrm{b}} \pm \\
53.23\end{array}$ & $\begin{array}{l}5268.71^{\mathrm{C}} \pm \\
50.97\end{array}$ \\
\hline $\begin{array}{l}\text { Total progressive motile sperm per } \\
\text { ejaculate (million) }\end{array}$ & $\begin{array}{l}2732.39^{\mathrm{a}} \pm \\
36.26\end{array}$ & $\begin{array}{l}2199.18^{\mathrm{b}} \pm \\
31.96\end{array}$ & $\begin{array}{l}2614.94^{\mathrm{C}} \pm \\
31.22\end{array}$ \\
\hline Post thaw sperm motility (\%) & $38.99^{a} \pm 0.41$ & $\begin{array}{l}35.55^{\mathrm{a}} \pm \\
0.49\end{array}$ & $\begin{array}{l}38.38^{\mathrm{a}} \pm \\
0.39\end{array}$ \\
\hline $\begin{array}{l}\text { Total post-thaw motile sperm per } \\
\text { ejaculate (million) }\end{array}$ & $\begin{array}{l}2537.51^{A} \pm \\
49.33^{A}\end{array}$ & $\begin{array}{l}1923.16^{\mathrm{B}} \pm \\
45.21\end{array}$ & $\begin{array}{l}2385.89^{C} \pm \\
43.99^{-}\end{array}$ \\
\hline $\begin{array}{l}\text { Frozen semen doses production } \\
(\mathrm{FSD}) / \text { ejaculate }\end{array}$ & $258.23^{a} \pm 4.99$ & $\begin{array}{l}214.33^{\mathrm{b}} \pm \\
5.45\end{array}$ & $\begin{array}{l}250.67^{\mathrm{a}} \pm \\
4.51\end{array}$ \\
\hline
\end{tabular}

Age of bulls

\begin{tabular}{|c|c|c|c|c|c|}
\hline Particulars & $\begin{array}{l}\text { Upto } 2 \text { years } \\
(\mathrm{n}=1666)\end{array}$ & $\begin{array}{l}2-3 \text { year }(n \\
=5991)\end{array}$ & $\begin{array}{l}3-4 \text { year }(n \\
=3922)\end{array}$ & $\begin{array}{l}4-5 \text { year }(n \\
=1810)\end{array}$ & $\begin{array}{l}\text { Above } 5 \text { year } \\
(n=939)\end{array}$ \\
\hline Ejaculate volume (ml) & $3.47^{a} \pm 0.04$ & $4.27^{b} \pm 0.02$ & $4.98^{c} \pm 0.03$ & $5.55^{d} \pm 0.05$ & $5.44^{d} \pm 0.07$ \\
\hline Concentration (million /ml) & $\begin{array}{l}892.80^{\mathrm{a}} \pm \\
13.92\end{array}$ & $\begin{array}{l}1029.63^{\mathrm{b}} \pm \\
7.42\end{array}$ & $\begin{array}{l}1115.55^{\mathrm{C}} \pm \\
9.27\end{array}$ & $\begin{array}{l}1243.93^{\mathrm{d}} \pm \\
12.82\end{array}$ & $\begin{array}{l}1295.68^{d} \pm \\
17.31\end{array}$ \\
\hline Progressive Motility (\%) & $35.85^{\mathrm{a}} \pm 0.55$ & $\begin{array}{l}40.13^{b} \pm \\
0.28\end{array}$ & $\begin{array}{l}48.79^{c} \pm \\
0.30\end{array}$ & $\begin{array}{l}52.79^{d} \pm \\
0.38\end{array}$ & $56.25^{\mathrm{e}} \pm 0.45$ \\
\hline SQI (0-100) & $42.76^{a} \pm 0.69$ & $\begin{array}{l}51.28^{\mathrm{b}} \pm \\
0.36\end{array}$ & $\begin{array}{l}62.51^{c} \pm \\
0.38\end{array}$ & $\begin{array}{l}70.45^{\mathrm{b}} \pm \\
0.48\end{array}$ & $74.29^{e} \pm 0.65$ \\
\hline $\begin{array}{l}\text { Total Sperm Output per ejaculate } \\
\text { (million) }\end{array}$ & $3226.11^{\mathrm{a}} \pm 68$ & $\begin{array}{l}4489.90^{\mathrm{b}} \pm \\
44.84\end{array}$ & $\begin{array}{l}5600.32^{C} \pm \\
59.34\end{array}$ & $\begin{array}{l}7058.29^{d} \pm \\
101.12\end{array}$ & $\begin{array}{l}7158.77^{d} \pm \\
135.08\end{array}$ \\
\hline $\begin{array}{l}\text { Total progressive motile sperm per } \\
\text { ejaculate (million) }\end{array}$ & $\begin{array}{l}1336.83^{\mathrm{a}} \pm \\
36.08\end{array}$ & $\begin{array}{l}2017.11^{\mathrm{b}} \pm \\
26.05\end{array}$ & $\begin{array}{l}2871.69^{c} \pm \\
36.18\end{array}$ & $\begin{array}{l}3798.10^{d} \pm \\
60.29\end{array}$ & $\begin{array}{l}4180.71^{\mathrm{e}} \pm \\
91.06\end{array}$ \\
\hline Post thaw sperm motility (\%) & $30.63^{a} \pm 0.82$ & $33.87^{b} \pm 0.4$ & $\begin{array}{l}39.81^{c} \pm \\
0.43\end{array}$ & $\begin{array}{l}42.32^{\mathrm{C}} \pm \\
0.58\end{array}$ & $46.82^{d} \pm 0.72$ \\
\hline $\begin{array}{l}\text { Total post-thaw motile sperm per } \\
\text { ejaculate (million) }\end{array}$ & $\begin{array}{l}1207.93^{\mathrm{a}} \pm \\
56.60^{-}\end{array}$ & $\begin{array}{l}1742.07^{\mathrm{b}} \pm \\
33.79\end{array}$ & $\begin{array}{l}2469.76^{\mathrm{C}} \pm \\
46.49\end{array}$ & $\begin{array}{l}3088.49^{d} \pm \\
72.63\end{array}$ & $\begin{array}{l}3908.81^{\mathrm{e}} \pm \\
120.58\end{array}$ \\
\hline
\end{tabular}

Row-wise means with different superscripts differ significantly $(a, b p<0.01, A, B p<0.05)$ 


\section{Seasons}

Frozen semen doses production (FSD)/ ejaculate

$151.32^{\mathrm{a}} \pm 8.8$

$194.73^{\mathrm{b}}+$

$245.76^{\mathrm{C}} \pm$ 4.31

4.58

$286.58^{\mathrm{d}} \pm$

6.72

$336.30^{\mathrm{e}} \pm 9.44$

Row-wise means with different superscripts differ significantly $(a, b p<0.01, A, B p<0.05)$

Table 4

Bull type $\mathrm{x}$ season interaction on various semen traits

\begin{tabular}{|lllllll|}
\hline Season & Summer & & Rainy & & Winter \\
\hline Bull Type & FSD & Non-FSD & FSD & Non-FSD & FSD & Non-FSD \\
\hline Ejaculate volume (ml) & $4.82 \pm$ & $4.23 \pm 0.06$ & $4.50 \pm$ & $4.33 \pm$ & $4.67 \pm$ & $4.58 \pm 0.06$ \\
& 0.03 & & 0.04 & 0.06 & 0.03 & \\
\hline Concentration (million /ml) & 1203.69 & $838.96 \pm$ & 1102.04 & $781.64 \pm$ & 1171.37 & $856.36 \pm$ \\
& \pm 9.65 & 17.61 & \pm 10.02 & 15.63 & \pm 8.57 & 15.08 \\
\hline Progressive Motility (\%) & $51.06 \pm$ & $23.15 \pm$ & $50.24 \pm$ & $26.31 \pm$ & $51.51 \pm$ & $25.48 \pm$ \\
& 0.28 & 0.58 & 0.32 & 0.57 & 0.27 & 0.51 \\
\hline SQI (0-100) & $65.71 \pm$ & $31.86 \pm$ & $62.97 \pm$ & $33.46 \pm$ & $65.82 \pm$ & $34.65 \pm$ \\
& 0.38 & 0.74 & 0.42 & 0.73 & 0.35 & 0.69 \\
\hline Total Sperm Output per ejaculate & 6001.03 & $3693.36 \pm$ & $5008.6 \pm$ & 3433.36 & 5633.34 & $4099.34 \pm$ \\
(million) & \pm 68.53 & 102.84 & 63.19 & \pm 90.06 & \pm 58.63 & 96.22 \\
\hline $\begin{array}{l}\text { Total progressive motile sperm per } \\
\text { ejaculate (million) }\end{array}$ & 3224.31 & $1041.33 \pm$ & 2626.35 & 1084.39 & $3036.9 \pm$ & $1261.67 \pm$ \\
\hline Post thaw sperm motility (\%) & \pm 41.81 & 43.14 & \pm 38.34 & \pm 41.81 & 36.16 & 44.24 \\
& $39.43 \pm$ & $17.83 \pm$ & $36.21 \pm$ & $19.57 \pm$ & $38.73 \pm$ & $17.68 \pm$ \\
\hline $\begin{array}{l}\text { Total post-thaw motile sperm per } \\
\text { ejaculate (million) }\end{array}$ & 0.41 & 1.69 & 0.49 & 1.53 & 0.39 & 1.83 \\
\hline
\end{tabular}

\subsection{Effect of age}

Age of bull showed very high significant $(\mathrm{P}<0.01)$ effect on VOL, SC, MOT, TSO, TSM, MOT-PT and TSM-PT. With increase in age all semen traits improved almost linearly. Semen VOL, SC, TSO enhanced significantly $(P<0.01)$ up to 5 years of age. Sperm progressive motility and post-thaw motility \% increased with age advancement and improved even beyond 5 years of age. Taking the base line TSO at 2 years old bulls, on annual basis the TSO increased by $39.17,73.59,118.77$ and $121.90 \%$ at 3,4,5 and > 5 years age, respectively; whereas, SC/ml increased by $15.33,24.94,39.33$ and $45.12 \%$, respectively. Thus, both increase in VOL and SC with advancement of age caused enhanced TSO with progress of age. Significant $(\mathrm{P}<$ 0.01) interaction between of bull type and age on VOL, SC, MOT, SQI, TSO, TSM indicated depending upon bull type semen quality improved significantly with age (Table 5). Age-season interaction on VOL, SC, MOT, SQI, TSO, TSM and TSM-PT indicated seasons effect was not similar $(P<0.01)$ in all the age groups of bulls (Table 6). 
Table 5

Age $\mathrm{X}$ bull type interaction on different semen characteristics

\begin{tabular}{|c|c|c|c|c|c|c|c|c|c|}
\hline Age & $\begin{array}{l}\text { Bull } \\
\text { Type }\end{array}$ & $\begin{array}{l}\text { Volume } \\
(\mathrm{ml})\end{array}$ & $\begin{array}{l}\text { Concentration } \\
\text { (million /ml) }\end{array}$ & $\begin{array}{l}\text { Progressive } \\
\text { motility (\%) }\end{array}$ & $\begin{array}{l}\text { SQI } \\
(0- \\
100)\end{array}$ & $\begin{array}{l}\text { Total } \\
\text { sperm } \\
\text { output } \\
\text { per } \\
\text { ejaculate } \\
\text { (million) }\end{array}$ & $\begin{array}{l}\text { Total } \\
\text { progressive } \\
\text { motile } \\
\text { sperm per } \\
\text { ejaculate } \\
\text { (million) }\end{array}$ & $\begin{array}{l}\text { Post- } \\
\text { thaw } \\
\text { motility } \\
(\%)\end{array}$ & $\begin{array}{l}\text { Total } \\
\text { post- } \\
\text { thaw } \\
\text { motile } \\
\text { sperm } \\
\text { per } \\
\text { ejaculate } \\
\text { (million) }\end{array}$ \\
\hline \multirow{2}{*}{$\begin{array}{l}\text { Up to } \\
2 \\
\text { years }\end{array}$} & FSD & $\begin{array}{l}3.33 \pm \\
0.05\end{array}$ & $\begin{array}{l}993.80 \pm \\
17.41\end{array}$ & $\begin{array}{l}44.66 \pm \\
0.65\end{array}$ & $\begin{array}{l}52.81 \\
\pm 0.82\end{array}$ & $\begin{array}{l}3404.23 \\
\pm 82.05\end{array}$ & $\begin{array}{l}1659.66 \pm \\
48.75\end{array}$ & $\begin{array}{l}31.38 \\
\pm 0.84\end{array}$ & $\begin{array}{l}1251.64 \\
\pm 58.81\end{array}$ \\
\hline & $\begin{array}{l}\text { Non- } \\
\text { FSD }\end{array}$ & $\begin{array}{l}3.67 \pm \\
0.07\end{array}$ & $\begin{array}{l}745.24 \pm \\
21.77\end{array}$ & $\begin{array}{l}22.97 \pm \\
0.70\end{array}$ & $\begin{array}{l}28.08 \\
\pm 0.93\end{array}$ & $\begin{array}{l}2965.9 \pm \\
116.13\end{array}$ & $\begin{array}{l}865.22 \pm \\
47.56\end{array}$ & $\begin{array}{l}17.25 \\
\pm 2.04\end{array}$ & $\begin{array}{l}434.23 \pm \\
74.13\end{array}$ \\
\hline \multirow[t]{2}{*}{$\begin{array}{l}2-3 \\
\text { Years }\end{array}$} & FSD & $\begin{array}{l}4.22 \pm \\
0.03\end{array}$ & $\begin{array}{l}1133.23 \pm \\
9.06\end{array}$ & $\begin{array}{l}48.75 \pm \\
0.29\end{array}$ & $\begin{array}{l}61.29 \\
\pm 0.39\end{array}$ & $\begin{array}{l}4889.2 \pm \\
56.43\end{array}$ & $\begin{array}{l}2508.9 \pm \\
33.6\end{array}$ & $\begin{array}{l}34.54 \\
\pm 0.41\end{array}$ & $\begin{array}{l}1773.44 \\
\pm 34.67\end{array}$ \\
\hline & $\begin{array}{l}\text { Non- } \\
\text { FSD }\end{array}$ & $\begin{array}{l}4.36 \pm \\
0.04\end{array}$ & $\begin{array}{l}844.41 \pm \\
11.84\end{array}$ & $\begin{array}{l}24.73 \pm \\
0.41\end{array}$ & $\begin{array}{l}33.37 \\
\pm 0.53\end{array}$ & $\begin{array}{l}3776.03 \\
\pm 71.27\end{array}$ & $\begin{array}{l}1137.88 \pm \\
33.26\end{array}$ & $\begin{array}{l}19.51 \\
\pm 1.20\end{array}$ & $\begin{array}{l}1073.43 \\
\pm 127.17\end{array}$ \\
\hline \multirow[t]{2}{*}{$\begin{array}{l}3-4 \\
\text { years }\end{array}$} & FSD & $\begin{array}{l}4.94 \pm \\
0.03\end{array}$ & $\begin{array}{l}1170.12 \pm \\
10.02\end{array}$ & $\begin{array}{l}52.66 \pm \\
0.28\end{array}$ & $\begin{array}{l}67.06 \\
\pm 0.37\end{array}$ & $\begin{array}{l}5835.04 \\
\pm 64.67\end{array}$ & $\begin{array}{l}3157.20 \pm \\
39.52\end{array}$ & $\begin{array}{l}39.92 \\
\pm 0.43\end{array}$ & $\begin{array}{l}2477.62 \\
\pm 46.57\end{array}$ \\
\hline & $\begin{array}{l}\text { Non- } \\
\text { FSD }\end{array}$ & $\begin{array}{l}5.21 \pm \\
0.09\end{array}$ & $\begin{array}{l}812.85 \pm \\
20.43\end{array}$ & $\begin{array}{l}27.37 \pm \\
0.74\end{array}$ & $\begin{array}{l}37.30 \\
\pm 0.93\end{array}$ & $\begin{array}{l}4298.16 \\
\pm 137.55\end{array}$ & $\begin{array}{l}1287.82 \pm \\
55.75\end{array}$ & $\begin{array}{l}13.33 \\
\pm 5.27\end{array}$ & $\begin{array}{l}723.49 \pm \\
367.25\end{array}$ \\
\hline \multirow[t]{2}{*}{$\begin{array}{l}4-5 \\
\text { years }\end{array}$} & FSD & $\begin{array}{l}5.57 \pm \\
0.05\end{array}$ & $\begin{array}{l}1246.8 \pm \\
13.02\end{array}$ & $53.7 \pm 0.37$ & $\begin{array}{l}71.34 \\
\pm 0.48\end{array}$ & $\begin{array}{l}7097.75 \\
\pm 103.85\end{array}$ & $\begin{array}{l}3865.03 \pm \\
61.75\end{array}$ & $\begin{array}{l}42.58 \\
\pm 0.58\end{array}$ & $\begin{array}{l}3106.04 \\
\pm 73.09\end{array}$ \\
\hline & $\begin{array}{l}\text { Non- } \\
\text { FSD }\end{array}$ & $\begin{array}{l}5.17 \pm \\
0.16\end{array}$ & $\begin{array}{l}1180.19 \pm \\
70.94\end{array}$ & $\begin{array}{l}32.63 \pm \\
1.95\end{array}$ & $\begin{array}{l}50.64 \\
\pm 2.82\end{array}$ & $\begin{array}{l}6182.16 \\
\pm 424.16\end{array}$ & $\begin{array}{l}2311.87 \pm \\
219.05\end{array}$ & $\begin{array}{l}17.14 \\
\pm 1.84\end{array}$ & $\begin{array}{l}1444.17 \\
\pm 162.36\end{array}$ \\
\hline \multirow{2}{*}{$\begin{array}{l}\text { Above } \\
5 \\
\text { years }\end{array}$} & FSD & $\begin{array}{l}5.44 \pm \\
0.07\end{array}$ & $\begin{array}{l}1295.68 \pm \\
17.31\end{array}$ & $\begin{array}{l}56.25 \pm \\
0.45\end{array}$ & $\begin{array}{l}74.29 \\
\pm 0.65\end{array}$ & $\begin{array}{l}7158.77 \\
\pm 135.08\end{array}$ & $\begin{array}{l}4180.71 \pm \\
91.06\end{array}$ & $\begin{array}{l}46.82 \\
\pm 0.72\end{array}$ & $\begin{array}{l}3908.81 \\
\pm 120.58\end{array}$ \\
\hline & $\begin{array}{l}\text { Non- } \\
\text { FSD }\end{array}$ & - & - & - & - & - & - & - & - \\
\hline
\end{tabular}


Table 6

Age $X$ season interaction on seminal parameters

\begin{tabular}{|c|c|c|c|c|c|c|c|c|c|}
\hline Age & Season & $\begin{array}{l}\text { Volume } \\
\text { (ml) }\end{array}$ & $\begin{array}{l}\text { Sperm } \\
\text { concentration } \\
\text { (million /ml) }\end{array}$ & $\begin{array}{l}\text { Progressive } \\
\text { motility (\%) }\end{array}$ & SQI & $\begin{array}{l}\text { TSO/ } \\
\text { ejaculate } \\
\text { (million) }\end{array}$ & $\begin{array}{l}\text { TSM/ } \\
\text { ejaculate } \\
\text { (million) }\end{array}$ & $\begin{array}{l}\text { MOT- } \\
\text { PT } \\
\text { motility } \\
(\%)\end{array}$ & $\begin{array}{l}\text { TSM- } \\
\text { PT/ } \\
\text { ejaculate } \\
\text { (million) }\end{array}$ \\
\hline \multirow{3}{*}{$\begin{array}{l}\text { Up to } \\
2 \\
\text { years }\end{array}$} & Summer & $\begin{array}{l}3.35 \pm \\
0.06\end{array}$ & $\begin{array}{l}912.28 \pm \\
23.21\end{array}$ & $\begin{array}{l}35.46 \pm \\
0.88\end{array}$ & $\begin{array}{l}42.53 \\
\pm 1.13\end{array}$ & $\begin{array}{l}3203.74 \\
\pm 109.89\end{array}$ & $\begin{array}{l}1336.81 \\
\pm 59.13\end{array}$ & $\begin{array}{l}31.29 \\
\pm 1.33\end{array}$ & $\begin{array}{l}1275.30 \\
\pm 97.34\end{array}$ \\
\hline & Rainy & $\begin{array}{l}3.49 \pm \\
0.07\end{array}$ & $\begin{array}{l}874.17 \pm \\
22.84\end{array}$ & $\begin{array}{l}36.12 \pm \\
0.90\end{array}$ & $\begin{array}{l}42.71 \\
\pm 1.13\end{array}$ & $\begin{array}{l}3158.53 \\
\pm 109.44\end{array}$ & $\begin{array}{l}1355.60 \\
\pm 60.22\end{array}$ & $\begin{array}{l}30.13 \\
\pm 1.27\end{array}$ & $\begin{array}{l}1162.25 \\
\pm 87.04\end{array}$ \\
\hline & Winter & $\begin{array}{l}3.62 \pm \\
0.08\end{array}$ & $\begin{array}{l}890.28 \pm \\
26.53\end{array}$ & $\begin{array}{l}36.04 \pm \\
1.11\end{array}$ & $\begin{array}{l}43.21 \\
\pm 1.34\end{array}$ & $\begin{array}{l}3368.99 \\
\pm 141.39\end{array}$ & $\begin{array}{l}1307.31 \\
\pm 68.98\end{array}$ & $\begin{array}{l}30.33 \\
\pm 1.80\end{array}$ & $\begin{array}{l}1168.37 \\
\pm 111.50\end{array}$ \\
\hline \multirow[t]{3}{*}{$\begin{array}{l}2-3 \\
\text { years }\end{array}$} & Summer & $\begin{array}{l}4.46 \pm \\
0.04\end{array}$ & $\begin{array}{l}1064.56 \pm \\
13.33\end{array}$ & $\begin{array}{l}40.28 \pm \\
0.49\end{array}$ & $\begin{array}{l}52.32 \\
\pm 0.62\end{array}$ & $\begin{array}{l}4889.26 \\
\pm 85.80\end{array}$ & $\begin{array}{l}2207.32 \\
\pm 49.79\end{array}$ & $\begin{array}{l}34.84 \\
\pm 0.65\end{array}$ & $\begin{array}{l}1896.36 \\
\pm 60.11\end{array}$ \\
\hline & Rainy & $\begin{array}{l}4.21 \pm \\
0.04\end{array}$ & $\begin{array}{l}995.64 \pm \\
12.80\end{array}$ & $\begin{array}{l}41.79 \pm \\
0.50\end{array}$ & $\begin{array}{l}52.18 \\
\pm 0.63\end{array}$ & $\begin{array}{l}4186.62 \\
\pm 71.70\end{array}$ & $\begin{array}{l}1926.37 \\
\pm 42.70\end{array}$ & $\begin{array}{l}33.46 \\
\pm 0.71\end{array}$ & $\begin{array}{l}1667.00 \\
\pm 55.22\end{array}$ \\
\hline & Winter & $\begin{array}{l}4.14 \pm \\
0.04\end{array}$ & $\begin{array}{l}1025.08 \pm \\
12.30\end{array}$ & $\begin{array}{l}38.54 \pm \\
0.48\end{array}$ & $\begin{array}{l}49.48 \\
\pm 0.62\end{array}$ & $\begin{array}{l}4363.92 \\
\pm 72.56\end{array}$ & $\begin{array}{l}1910.50 \\
\pm 41.62\end{array}$ & $\begin{array}{l}33.20 \\
\pm 0.71\end{array}$ & $\begin{array}{l}1643.93 \\
\pm 59.11\end{array}$ \\
\hline \multirow[t]{3}{*}{$\begin{array}{l}3-4 \\
\text { years }\end{array}$} & Summer & $\begin{array}{l}5.05 \pm \\
0.06\end{array}$ & $\begin{array}{l}1181.46 \pm \\
17.66\end{array}$ & $\begin{array}{l}50.26 \pm \\
0.53\end{array}$ & $\begin{array}{l}64.81 \\
\pm 0.69\end{array}$ & $\begin{array}{l}6088.51 \\
\pm 116.92\end{array}$ & $\begin{array}{l}3233.12 \\
\pm 72.52\end{array}$ & $\begin{array}{l}42.65 \\
\pm 0.71\end{array}$ & $\begin{array}{l}2889.87 \\
\pm 87.52\end{array}$ \\
\hline & Rainy & $\begin{array}{l}5.02 \pm \\
0.06\end{array}$ & $\begin{array}{l}1071.61 \pm \\
18.03\end{array}$ & $\begin{array}{l}46.38 \pm \\
0.60\end{array}$ & $\begin{array}{l}59.68 \\
\pm 0.77\end{array}$ & $\begin{array}{l}5327.11 \\
\pm 112.94\end{array}$ & $\begin{array}{l}2610.21 \\
\pm 66.74\end{array}$ & $\begin{array}{l}37.56 \\
\pm 0.97\end{array}$ & $\begin{array}{l}2198.01 \\
\pm 93.19\end{array}$ \\
\hline & Winter & $\begin{array}{l}4.90 \pm \\
0.05\end{array}$ & $\begin{array}{l}1093.35 \pm \\
13.46\end{array}$ & $\begin{array}{l}49.13 \pm \\
0.45\end{array}$ & $\begin{array}{l}62.49 \\
\pm 0.57\end{array}$ & $\begin{array}{l}5405.70 \\
\pm 84.26\end{array}$ & $\begin{array}{l}2761.69 \\
\pm 51.12\end{array}$ & $\begin{array}{l}38.82 \\
\pm 0.64\end{array}$ & $\begin{array}{l}2294.85 \\
\pm 64.59\end{array}$ \\
\hline \multirow[t]{3}{*}{$\begin{array}{l}4-5 \\
\text { years }\end{array}$} & Summer & $\begin{array}{l}5.65 \pm \\
0.09\end{array}$ & $\begin{array}{l}1293.31 \pm \\
24.05\end{array}$ & $\begin{array}{l}52.84 \pm \\
0.70\end{array}$ & $\begin{array}{l}70.98 \\
\pm 0.88\end{array}$ & $\begin{array}{l}7447.51 \\
\pm 187.64\end{array}$ & $\begin{array}{l}4026.08 \\
\pm 109.45\end{array}$ & $\begin{array}{l}44.02 \\
\pm 0.98\end{array}$ & $\begin{array}{l}3404.83 \\
\pm 135.27\end{array}$ \\
\hline & Rainy & $\begin{array}{l}5.38 \pm \\
0.10\end{array}$ & $\begin{array}{l}1139.98 \pm \\
24.22\end{array}$ & $\begin{array}{l}53.14 \pm \\
0.76\end{array}$ & $\begin{array}{l}68.95 \\
\pm 0.97\end{array}$ & $\begin{array}{l}6201.25 \\
\pm 189.27\end{array}$ & $\begin{array}{l}3362.59 \\
\pm 119.54\end{array}$ & $\begin{array}{l}41.13 \\
\pm 1.32\end{array}$ & $\begin{array}{l}2751.10 \\
\pm 159.88\end{array}$ \\
\hline & Winter & $\begin{array}{l}5.57 \pm \\
0.06\end{array}$ & $\begin{array}{l}1261.61 \pm \\
18.94\end{array}$ & $\begin{array}{l}52.59 \pm \\
0.55\end{array}$ & $\begin{array}{l}70.82 \\
\pm 0.71\end{array}$ & $\begin{array}{l}7215.92 \\
\pm 150.83\end{array}$ & $\begin{array}{l}3858.09 \\
\pm 89.47\end{array}$ & $\begin{array}{l}41.85 \\
\pm 0.84\end{array}$ & $\begin{array}{l}3059.59 \\
\pm 100.00\end{array}$ \\
\hline \multirow{3}{*}{$\begin{array}{l}\text { Above } \\
5 \\
\text { years }\end{array}$} & Summer & $\begin{array}{l}5.70 \pm \\
0.11\end{array}$ & $\begin{array}{l}1354.04 \pm \\
26.81\end{array}$ & $\begin{array}{l}55.96 \pm \\
0.74\end{array}$ & $\begin{array}{l}76.19 \\
\pm 1.01\end{array}$ & $\begin{array}{l}7853.33 \\
\pm 232.82\end{array}$ & $\begin{array}{l}4541.93 \\
\pm 155.25\end{array}$ & $\begin{array}{l}45.97 \\
\pm 1.30\end{array}$ & $\begin{array}{l}4262.88 \\
\pm 208.09\end{array}$ \\
\hline & Rainy & $\begin{array}{l}5.25 \pm \\
0.16\end{array}$ & $\begin{array}{l}1077.69 \pm \\
38.66\end{array}$ & $\begin{array}{l}52.66 \pm \\
1.26\end{array}$ & $\begin{array}{l}66.18 \\
\pm 1.77\end{array}$ & $\begin{array}{l}5740.90 \\
\pm 275.17\end{array}$ & $\begin{array}{l}3150.93 \\
\pm 172.77\end{array}$ & $\begin{array}{l}46.48 \\
\pm 2.18\end{array}$ & $\begin{array}{l}2896.32 \\
\pm 276.11\end{array}$ \\
\hline & Winter & $\begin{array}{l}5.29 \pm \\
0.09\end{array}$ & $\begin{array}{l}1324.49 \pm \\
26.47\end{array}$ & $\begin{array}{l}57.75 \pm \\
0.63\end{array}$ & $\begin{array}{l}75.59 \\
\pm 0.92\end{array}$ & $\begin{array}{l}7088.66 \\
\pm 194.57\end{array}$ & $\begin{array}{l}4246.88 \\
\pm 134.76\end{array}$ & $\begin{array}{l}47.45 \\
\pm 0.93\end{array}$ & $\begin{array}{l}3894.47 \\
\pm 165.80\end{array}$ \\
\hline
\end{tabular}

TS = Total Sperm; TSM = Total motile sperm; MOT-PT = Post thaw motility; TSM-PT: Total motile sperm -Post thaw

Overall basis, semen quality index (SQI) increased steadily $(\mathrm{P}<0.01)$ from 2 to $>5$ years of age (Fig. 1) in FSD bulls, where as in Non-FSD bulls' increments were negligible. Like TSO, TSM-PT also increased by 1.42, 1.98, 2.48 and 3.12 times from base value at 2-year age to $3 \mathrm{rd}, 4$ th, 5 th and $>5$ years of age, respectively in FSD bulls (Table 5). Production of FSD / ejaculate was significantly $\left(\mathrm{F}_{4,2385}=82.47, \mathrm{P}<0.01\right)$ increased with age (Table 5$)$ and it increased by 2 folds at 60 months as compared to their FSD production levels at 24 months age. Significant interaction effect $\left(F_{8,2385}=2.61, P<0.01\right)$ of age $X$ season on FSD production / ejaculates indicated depending on seasons, FSD production varied significantly with age of 
bulls (Fig. 2). The coefficients of correlation ( $r$ ) between age and seminal attributes are illustrated in Table 7. Age of bulls showed positive correlation $(\mathrm{P}<0.01)$ with all semen parameters indicated improvement in semen traits with age of bulls.

Table 7

Interrelationship betweenage (months) andsemen parameters of crossbred dairy bulls

\begin{tabular}{|c|c|c|c|}
\hline Particulars & $\begin{array}{l}\text { Correlation } \\
\text { coefficient } \\
\text { (r) }\end{array}$ & Particulars & $\begin{array}{l}\text { Correlation coefficient } \\
\text { (r) }\end{array}$ \\
\hline Volume (ml) & $0.30 * *$ & Total sperm output per ejaculate (million) & $0.31^{* \star}$ \\
\hline $\begin{array}{l}\text { Concentration (million } \\
/ \mathrm{ml} \text { ) }\end{array}$ & $0.19 * \star$ & $\begin{array}{l}\text { Total progressive motile sperm per ejaculate } \\
\text { (million) }\end{array}$ & $0.36 * \star$ \\
\hline $\begin{array}{l}\text { Progressive motility } \\
(\%)\end{array}$ & $0.29 * \star$ & $\begin{array}{l}\text { Total post-thaw motile sperm per ejaculate } \\
\text { (million) }\end{array}$ & $0.42^{* *}$ \\
\hline Post thaw motility (\%) & $0.28 * \star$ & $\begin{array}{l}\text { Frozen semen doses production (FSD)/ } \\
\text { ejaculate }\end{array}$ & $0.38 * \star$ \\
\hline SQI (0-100) & $0.34^{\star \star}$ & & \\
\hline
\end{tabular}

\subsection{Effect of ejaculation frequency}

Statistical analysis revealed significant $(\mathrm{P}<0.01)$ variations in semen quality between first and second ejaculates viz. VOL, SC, MOT, TSO, TSM, MOT-PT and TSM-PT. First ejaculates had higher $(\mathrm{P}<0.01)$ VOL, SC, TSO and TSM-PT and lower MOT, TSM and MOT-PT as compared to successive (second) ejaculates. The SC, TSO and TSM-PT were 14.40, 16.30 and 5.92\% less in 2nd ejaculates. However, overall SQI did not differ between between 1st and 2nd ejaculates. Frequency of collection significantly $\left(F_{1,2385}=201.37, P<0.01\right)$ affected FSD production / ejaculate. Frozen semen doses production was $32.69 \%$ higher in first ejaculates as compared to second ejaculates (Table 2).

\section{Discussion}

Frozen semen doses (FSD) production centers select semen samples for cryopreservation based on semen volume, sperm concentration, sperm kinetics and morphology. Thus, factors that affect initial semen quality play very deterministic roles on selection of ejaculates for processing, and it subsequently influence freezability, post-thaw motility, FSD production, sperm fertility and ultimately success of Al and embryo production. A wide range of factors that affect seminal attributes have been reported viz. breed, age, body condition score, testicular morphology, vascularity of testicular cones, testicular thermo-regulation, gonadal development, thero-adaptability of bulls etc. (Brito et al. 2002a,b; Mandal et al. 2004, 2009, 2010; Kastelic, 2013; Kastelic et al. 2018). Although problems of cryo-preservability, high reproductive wastage rate etc. are major issues with B. taurus X B. indicus breeding programmes (Mandal et al. 2012; Singh et al. 2013), however, the same has been inadequately addressed in tropical countries. Present investigation provides a detailed account of important factors affecting reproductive ability in crossbred dairy bulls.

Values (mean \pm SE) of seminal attributes of HF X Sahiwal dairy bulls observed in this study were within the normal range as reported earlier HF X Sahiwal crossbred bulls (Mandal et al. 2009, 2010) and other HF crossbreds bulls (Chauhan et al. 2010). Present findings indicated that semen quality improved gradually with age (Table 1). In HF X Sahiwal crossbred bulls sperm concentration increased significantly up to 5th year of age, thereafter increase was marginal and nonsignificant. This was major difference between presently studied HF crossbred bulls and exotic HF bulls, where sperm concentration reported to increase 1-3 years of age (Taylor et al. 1985; Argiris et al. 2018) in pure HF bulls. In zebu bulls maturity occurs at $\geq 4$ years (Addass et al. 2011; Bhakat et al. 2011) because of slow body development and gradual 
attainment of sexual maturity. This might be the same reason for improvement in sperm productivity with age in tauruszebu crossbred bulls (Mandal et al. 2004; Addass et al. 2011). Improvement in semen quality with age could be attributed to sexual and gonadal development, increase in testicular circumference, numbers of seminiferous tubules, enhancement in size of testes, maturity and adequate development of testes vascular cone and other apparatus responsible for effective thermo-regulation of testes etc. which in combination influences spermatogenesis, sperm production capacity and over all semen quality depending upon breeds and species involved (Brito et al. 2002a, b; Mandal et al. 2004; Sonj et al. 2013). Impact of seasonal heat stress on sperm productivity greatly varied with breeds and it also depends upon individual capacity of heat tolerance. In heat tolerant Frieswal (HF X Sahiwal) bulls the growth rate, body and testicular development, and semen production performance was better than less heat tolerant bulls (Mandal and Tyagi, 2009). Heat tolerance capacities of zebu were more than taurus bulls and produce less abnormal sperms under stressful conditions (Koivisto et al. 2009).

In Al programme number of bulls to be reared in FSD production centers depends upon sperm production ability of bulls and cryo-sustainability of semen. In this study, a large number of crossbred bulls $(50.56 \% ; 136$ of 269$)$ did not produced any FSD and ultimately rejected from breeding programme although they were physically sound during their induction to collection programme at 15-18 months of age. In purebred bulls, reproductive wastages were comparatively less as compared to crossbred bulls (Chacko, 2005; Singh et al. 2013) and therefore, quality semen productions from crossbred bulls remained as a challenging issue to breed large crossbred cow population in tropical countries. In fact, crossbred cows are the major contributors to total cow milk production in most of the tropical countries and there are steady requirements of quality FSDs of crossbred bulls. However, as revealed in the present study, in some selected FSD bulls (Table 5), improvement and maintenance of quality even beyond 5 years of age (maximum age of bulls was 93 months in this study) indicated existence of potentials for high sperm production in selective HF X Sahiwal dairy crossbred bulls, which needs selection, maintenance and further propagation.

In Non-FSD bulls, although volume and concentration increased with age (Table 5), however, sperm kinematics (MOT) did not improve much with age. In these bulls, the overall SQI showed very marginal improvement (Fig. 1) with age, such type of bulls had no practical breeding utility in FSD production centers. The findings indicated that there was wide difference in SQI even at 2-years of age between FSD (SQI = 52.81) and Non-FSD bulls (SQI = 28.08). In Non-FSD bulls age had no significant impact on subsequent SQI improvement even up to 4 years of age reared in this production stations (Fig. 1). Present results suggested that young bulls having $\mathrm{SQI}<50$ should be monitored precisely in FSD production centers and need culling as early as possible, even at 24 months age to avoid unnecessary expenditures and economic losses. In an earlier study (Mandal et al. 2010), it was recommended for culling of bulls $<30$ months age based on sperm morphology, motility and concentration. However, present study denote for culling bulls at 24 months age provided SQI $<50$. Improvement in SQI in few Non-FSD bulls between 4-5 years of age was due to some mediocre bulls that remained in borderlines of semen freezability. In these bulls, it is very difficult to be ascertained about their continuation in FSD production centers, because they produce inconsistent semen quality, ejaculates with incoherent freezability and post-thaw motility. Similar problems also arise on determination of bull fertility either in Al or natural insemination, where differentiation between excellent and poor (infertile) bull is easier than the sub-fertile ones and there exists considerable knowledge gaps in identification of sub-fertile bulls (Kastelic, 2013).

Highly significant $(P<0.01)$ positive association of age with seminal (Table 7$)$ attributes indicated improvement in semen traits with advancement of age. Significant positive correlation among semen traits indicated for collateral improvement in these traits, if selection pressure is applied for any one of the important traits like age, age of semen donation, early age of puberty, age of freezable quality semen production etc.

Seasons' effect is the outcome of a very complex interaction of animal system with various environmental variables like temperature, humidity, sunshine, photoperiod, precipitation etc. that directly influence the animals and indirectly via alteration in seasonal fodder and feed quality. The study location had dry hot summer, very hot-humid rainy and

Page 13/19 
moderately cold-humid winter seasons; variations in maximum temperature $9-46^{\circ} \mathrm{C}$, minimum temperature $2-31^{\circ} \mathrm{C}$ and relative humidity 30-100\% (Mandal et al. 2002). Bulls in the present study had the lowest SQI in rainy season and FSD production per ejaculate was also $16-20 \%$ less in this season. As the process of spermatogenesis is very lengthy one (6062 days in bull), the seasonal influence affect both meiotic and spermiogenesis stages of spermatogenesis and its impact is long lasting and carried forward. Qualitative deterioration of semen, less production of frozen semen doses in hot-humid rainy seasons as observed in the present study signifies importance of seasons in bull station and FSD production units. From the regions of temperate climatic conditions also, decrease in sperm quality due to exposure of bulls to natural heat stress has recently been reported (Sabés-Alsina et al. 2017; Luceño et al. 2020)

Because of direct visible impact of seasons on milk production, seasonality on production traits had been extensively studied in lactating cattle (Kadzere et al. 2002; Dash et al. 2016; Mandal et al. 2021a,b). As milk production directly linked with calf production, studies on reproductive physiology of females, oocyte production, embryo development, fertilization etc. had been given more research emphasis and this disparity lead more research focus on female, ignoring males (Roth, 2017; Luceño et al. 2020). The physiological impact of meteorological factors viz. temperature, humidity and seasonality as a whole on the bull has not been established like that of female part and the molecular mechanisms of sperm damage owing to environmental stress have not been entirely characterized (Rahman et al. 2014; Luceño et al. 2020).

As stated earlier, the major aim of any bull station is to produce maximum numbers of quality frozen semen doses (FSD). Present study showed qualitative deterioration in semen characteristics and quantitative reduction (14-20\%) of FSD/ejaculate during rainy season and definitely this reduction have substantial financial impacts on FSD production stations. Ejaculates collected in rainy season (July-October) basically had the spermatozoa synthesized and matured at least 60 days before, the period coincides with long duration day length (21st June, longest day in northern hemisphere). Higher duration of photoperiod and longer day length affect semen quality (Sonj et al. 2013; Granados et al. 2014) through melatonin modulated spermatogenesis, maturation and other sperm functions (González-Arto et al. 2014; Fernández-Alegre et al. 2020). Thus, combined effect of high temperature, humidity, photoperiod and other indirect seasonal effects mediated through feed quality might be responsible for semen quality deterioration in rainy season in this study. The present investigation also revealed that in Non-FSD bulls, SQI did not improve after 2 years of age and SQI value remained $<50$ (33.40 \pm 0.42 ). Therefore, bulls' SQI $<50$ at 2 years age need immediate culling to avert financial losses because such bulls are non-productive for artificial breeding and inflict an economic burden (estimated to Rupees 73,000 /bull/ year) for maintenance in the present Bull Rearing Unit. Although these bulls are of high genetic merit, received after nominated mating between high yielding dams $x$ proven sires and passed through two stage selection process (at 3-6 months based on body morphometric characteristics and 12-15 month, breeding soundness evaluation), still the high rejection rate $(50.56 \%, 136$ of 269 in this study) of crossbred bulls is a very serious issue (Chacko, 2005; Tyagi et al. 2006; Mandal et al. 2012; Singh et al. 2013) in crossbreeding programmes in tropical countries. Mating of B. taurus $x$ B. indicus is commonly termed as crossbreeding, but practically it is an inter-species mating, which might have accentuated the problems of poor reproductive ability in crossbred bulls (Singh et al. 2013). Present finding suggested that age is one the most important factors affecting semen quality in HF X Sahiwal crossbred bulls; sperm productivity in FSD bulls increased even 5 years of age (unlike up to 3 years age in exotic HF bulls) which need selection for improvement in semen quality traits. Summer and winter seasons should be effectively used for optimum FSD production from crossbred bulls maintained under similar climatic conditions. Bulls having SQI $<50$ need to be culled at 2 years of age without further expectation on improvement in semen quality. Findings of the present study recommended that thermal stress amelioration measures should be taken up at bull stations from late summer to rainy seasons to minimize negative impacts of environment.

\section{Conclusions}

The study concluded that management factors such as age of bulls, collection season, collection frequency, bull type significantly affected the overall semen quality, sperm productivity, cryo-preservation capacity of ejaculates and FSD production in HF X Sahiwal crossbred bulls. Depending upon season, qualitative and quantitative attributes of semen

Page 14/19 
improved with advancement of age, being substantial in FSD and negligible in Non-FSD bulls. In Non-FSD bulls' semen quality do not improve beyond 2-years age and bulls having $\mathrm{SQI}<50$ need immediate culling for economic reasons.

\section{Declarations}

Acknowledgement: Authors are thankful to Director, ICAR-Central Institute for Research on Cattle, Meerut (Formerly Project Directorate on Cattle, Meerut) for providing necessary facilities and funds

Statement of Animal Rights: Not applicable

Conflict of interest/Competing interests: The authors in this study declare that they have no conflict of interest.

Funding:Work carried out under routine institution funds through 'Frieswal Project'

Ethics approval: Not applicable, as semen collection from bulls was a routine job for Al centre

Consent to participate: Agreed

Consent for publication: All the authors agree for publication of the article, if accepted for publication

Availability of data and material: No data will be available for others use

Code availability (software application or custom code): Not applicable

Authors' contributions: DKM: Analyzed data and wrote the manuscript, DKM, MK, ST: All 3 authors contributed equally to research works, semen evaluation, cryo-preservation and execution of works.

\section{References}

1. Addass, P. A., 2011. Effect of age and body condition score on sperm production potential among some indigenous bull cattle in Mubi Adamawa State, Nigeria. Agriculture and Biology Journal of North America, 2, 203-206.

2. Argiris, A., Ondho, Y. S., Santoso, S. I., Kurnianto, E., 2018. Effect of age and bull on fresh semen quality and frozen semen production of Holstein bulls in Indonesia. IOP Conf. Series: Earth and Environmental Science, 119, 012033 (doi :10.1088/1755-1315/119/1/012033).

3. Argov, N., Sklan, D., Zeron, Y., Roth, Z., 2007. Association between seasonal changes in fatty-acid composition, expression of VLDL receptor and bovine sperm quality. Theriogenology, 67, 878-885.

4. Argov-Argaman, N., Mahgrefthe, K., Zeron, Y., Roth, Z., 2013. Season-induced variation in lipid composition is associated with semen quality in Holstein bulls. Reproduction, 145, 479-489.

5. Barth, A. D., Oko, R. J., 1989. Abnormal morphology of bovine spermatozoa. lowa State University Press, lowa.

6. Bhakat, M., Mohanty, T. K., Raina, V. S., Gupta, A. K., Khan, H. M., Mahapatra, R .K., Sarkar, M., 2011. Effect of age and season on semen quality parameters in Sahiwal bulls. Tropical Animal Health and Production, 43, 1161-1168.

7. Brito, L. F .C., Silva, A. E. D. F., Rodrigues, L. H., Vieira, F. V., Deragon, L. A. G., Kastelic, J. P., 2002a. Effects of environmental factors, age and genotype on sperm production and semen quality in Bos indicus and Bos taurus Al bulls in Brazil. Animal Reproduction Science, 70, 181-190.

8. Brito, L. F. C., Silva, A. E. D. F., Rodrigues, L. H., Vieira, F. V., Deragon, L. A. G., Kastelic, J. P., 2002b. Effect of age and genetic group on characteristics of the scrotum, testes and testicular vascular cones, and on sperm production and semen quality in Al bulls in Brazil. Theriogenology, 58, 1175-1186.

9. Chacko, C. T., 2005. Development of the Sunandini cattle breed in India. Retrieved from http://www.fao.org/livestock/agap/war/warall/t4650b/t4650b0v.htm

Page 15/19 
10. Chauhan, I. S., Gupta, A. K., Khate, K., Chauhan, A., Rao, T. K. S., Pathak, S., Hazra, R., Singh, M., 2010. Genetic and non-genetic factors affecting semen production traits in Karan Fries crossbred bulls. Tropical Animal Health and Production, 42, 1809-1815.

11. Dash, R., Sailo, L., Verma, N., Bharti, P., Saikia, J., Imtiwati., Kumar, R., 2016. Impact of heat stress on health and performance of dairy animals: A review. Veterinary World, 9, 260-268.

12. Everett, R. W., Bean, B., 1982. Environmental influences on semen output. Journal of Dairy Science, 65, 1303-1310.

13. Fernández-Alegre, E., Álvarez-Fernández, I., Domínguez, J.C., Casao, A., Martínez-Pastor, F. 2020. Melatonin NonLinearly Modulates Bull Spermatozoa Motility and Physiology in Capacitating and Non-Capacitating Conditions. International Journal of Molecular Science, 21, 2701, doi:10.3390/ijms21082701

14. González-Arto, M., Vicente-Carrillo, A, Martínez-Pastor, F., Fernández-Alegre, E., Roca, J., Miró, J., Rigau, T., RodríguezGil, J. E., Pérez-Pé, R., Muiño-Blanco, T., Cebrián-Pérez, J. A., Casao, A. 2014. Melatonin receptors MT1 and MT2 are expressed in spermatozoa from several seasonal and nonseasonal breeder species. Theriogenology, 86, 1958-1968.

15. Granados, M. D. G., López, L. E. H., Aguilar, A. C., Molina, A. L. C., Pérez-Ramírez, O., Mondragón-Ceballos, R. 2014. Effect of photoperiod on characteristics of semen obtained by electroejaculation in stump-tailed macaques (Macaca arctoides). Primates, 55, 393-401.

16. Hafez, E. S. E., 1993. Reproduction in Farm Animals. $6^{\text {th }}$ Edition, Lea Febiger, Philadelphia, USA.

17. Kadzere, C. T., Murphy, M. R., Silanikove, N., Maltz, E. 2002. Heat stress in lactating dairy cows: a review. Livestock Production Science, 77, 59-91.

18. Kastelic, J. P., 2013. Male involvement in fertility and factors affecting semen quality in bulls. Anim. Front. 3, 20-25. doi:10.2527/af.2013-0029 (Downloaded from https://academic.oup.com/af/article/3/4/20/4638651 by InterUniversity Centre for Astronomy and Astrophysics user on 22 February 2021).

19. Kastelic, J. P., Rizzoto, G., Thundathil, J., 2018. Review: Testicular vascular cone development and its association with scrotal thermoregulation, semen quality and sperm production in bulls. Animal, 12, S1, pp s133-s141 (doi:10.1017/S1751731118001167).

20. Koivisto, M. B., Costa, M. T. A., Perri, S. H. V., Vicente, W. R. R., 2009. The effect of season on semen characteristics and freezability in Bos indicus and Bos taurus bulls in the southeastern region of Brazil. Reproduction in Domestic Animals, 44, 587-592.

21. Luceño N. L., Angrimani, D. S. R., Bicudo, L. C., Szymańska, K. J., Poucke M. V., Demeyere, K., Meyer, E., Peelman, L., Mullaart, E., Broekhuijse, M. L. W. J., Soom, A. V., 2020. Exposing dairy bulls to high temperature-humidity index during spermatogenesis compromises subsequent embryo development in vitro. Theriogenology, 141, 16-25.

22. Mandal, D. K., 1998. Effect of surface cooling on reproductive performance of murrah buffalo bulls. Ph.D. Thesis. ICARNational Dairy Research Institute, Karnal, India.

23. Mandal, D. K., Bhaka,t C., Dutta, T. K., 2021b. Impact of environmental factors on physiological adaptability, thermotolerance indices, and productivity in Jersey crossbred cows. International Journal of Biometeorology, https://doi.org/10.1007/s00484-021-02157-2.

24. Mandal, D. K., Kumar, M., Tyagi, S., 2010. Effect of age on spermiogram of Holstein Friesian $\times$ Sahiwal crossbred bulls. Animal, 4, 595-603.

25. Mandal, D. K., Kumar, M., Tyagi, S., Ganguly, I., Kumar, S., Gaur, G. K., 2012. Patterns of reproductive wastage and inheritance of semen quality in Frieswal crossbred bulls. Tamil Nadu Journal of Veterinary and Animal Science, 8, 245249.

26. Mandal, D. K., Mandal, A., Bhakat, C., Dutta, T. K., 2021a. Effect of heat stress amelioration through open-ridge ventilated thatched roof housing on production and reproduction performance of crossbred Jersey cows. Tropical Animal Health and Production, 53, 144. https://doi.org/10.1007/s11250-021-02574-w 
27. Mandal, D. K., Nagpaul, P. K., Gupta, A. K., 2003. Motion characteristics of Murrah buffalo bulls spermatozoa in various seasons and its relationship with functional integrity of the plasmallema. Theriogenology, 60, 349-358.

28. Mandal, D. K., Rao, A. V. M. S., Singh, K., Singh, S. P., 2002. Comfortable macroclimatic conditions for optimum milk production in Sahiwal cows. Journal of Applied Zoological Research, 13, 228-230.

29. Mandal, D. K., Singh, K., Tyagi, S., 2004. Age related changes in body size and gonadal development of growing Frieswal bulls. Indian Journal of Animal Sciences, 74, 31-34

30. Mandal, D.K., Tyagi, S., 2009. Body growth, gonadal development and semen quality of Frieswal bulls in relation to heat tolerance. Indian Veterinary Journal, 86, 356-359.

31. Morrell, J. M., 2020. Heat stress and bull fertility. Theriogenology, 153, 62-67.

32. Orgal, S., Zeron, Y., Elior, N., Biran, D., Friedman, E., Druker, S., Roth, Z., 2012. Season-induced changes in bovine sperm motility following a freeze-thaw procedure. Journal of Reproduction and Development, 58, 212-218.

33. Rahman, M. B., Vandaele, L., Rijsselaere, T., El-Deen, M. S., Maes, D., Shamsuddin, M., Soom, A. V., 2014. Bovine spermatozoa react to in vitro heat stress by activating the mitogen-activated protein kinase 14 signalling pathway. Reproduction Fertility and Development, 26, 245-257.

34. Roth, Z., 2017. Effect of heat stress on reproduction in dairy cows: insights into the cellular and molecular responses of the oocyte. Annual Review of Animal Biosciences, 5, 151-170.

35. Sabés-Alsina, M., Lundeheim, N., Johannisson, A., Lopez-Bejar, M., Morrell, J.M., 2019. Relationships between climate and sperm quality in dairy bull semen: a retrospective analysis. Journal of Dairy Science, 102, 1-11.

36. Sabés-Alsina, M., Johannisson, A., Lundeheim, N., Lopez-Bejar, M., Morrell, J. M., 2017. Effects of season on bull sperm quality in thawed samples in northern Spain. Veterinary Records, 180, 251 (doi: 10.1136/vr.103897.).

37. Singh, S. P., Mandal, D. K., Tyagi, S., Mathur, A. K., 2013. Reproductive performance of crossbred bulls in tropics-The Indian Experience. ibdc Publishers, 7 Meerabai Marg, Lucknow-226001, Uttar Pradesh, India.

38. Snedecor, G. W., Cochran, W. G., 1994. Statistical Methods. 8th Edition, Oxford and IBH Publishing Co., New Delhi.

39. Snoj, T., Kobal, S., Majdic, G., 2013. Effects of season, age, and breed on semen characteristics in different Bos taurus breeds in a 31-year retrospective study. Theriogenology, 79, 847-852.

40. SPSS Inc. Released, 2007. SPSS for Windows, Version 16.0. Chicago, SPSS Inc.

41. Taylor, J.F., Bean, B. Marshall, C. E., Sullivan, J. J., 1985. Genetic and environmental components of semen production traits of artificial insemination Holstein bulls. Journal of Dairy Science, 68, 2703-2722.

42. Tyagi, S., Mandal, D. K., Kumar, M., Mathur, A. K., 2006. Reproductive wastage rate of crossbred dairy bulls with reference to level of exotic inheritance and number of breed components. Indian Journal of Animal Reproduction, 27, 27-30.

\section{Figures}




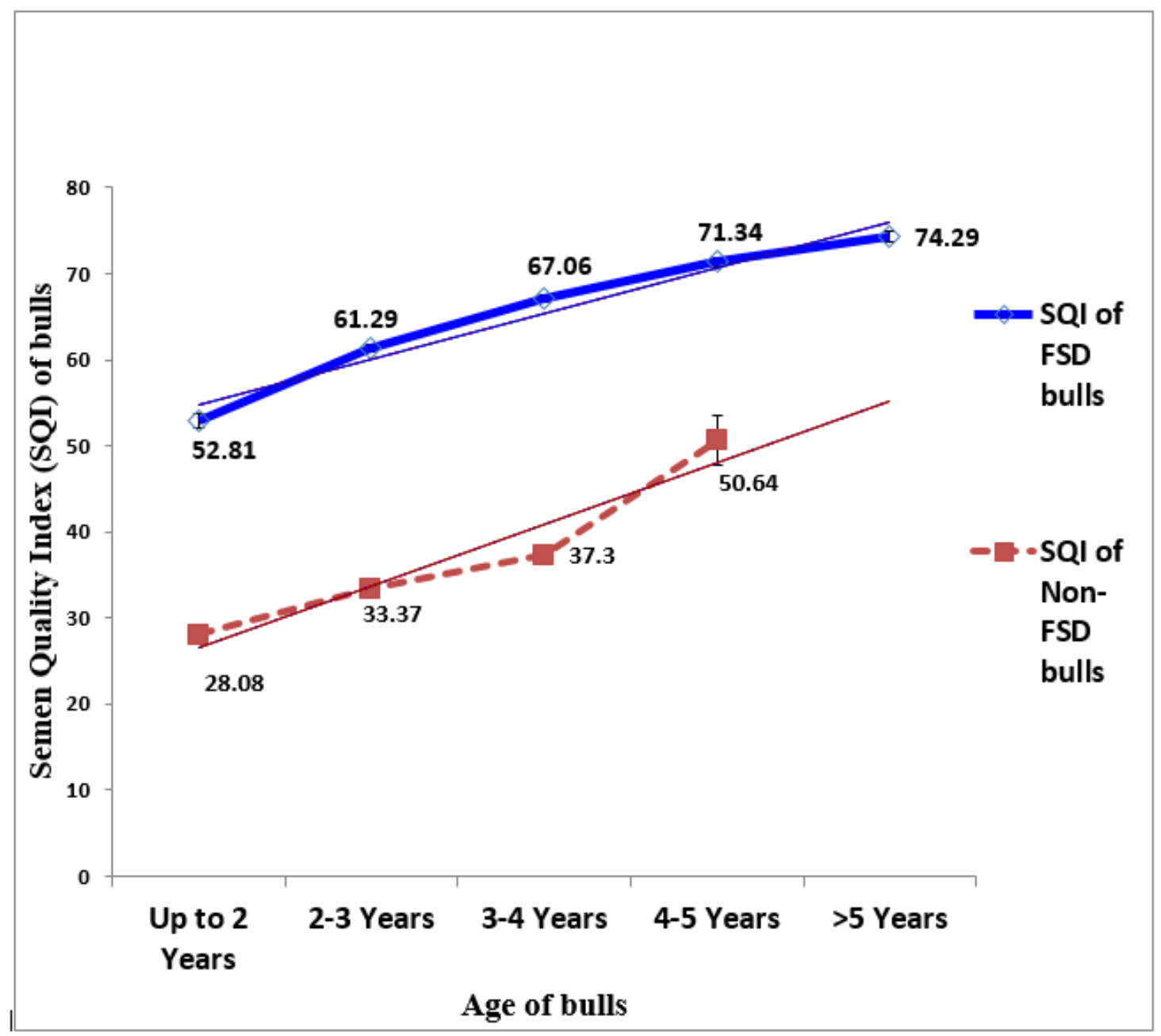

Figure 1

Semen Quality Index (SQI) of Non-FSD and FSD producer bulls over the age 


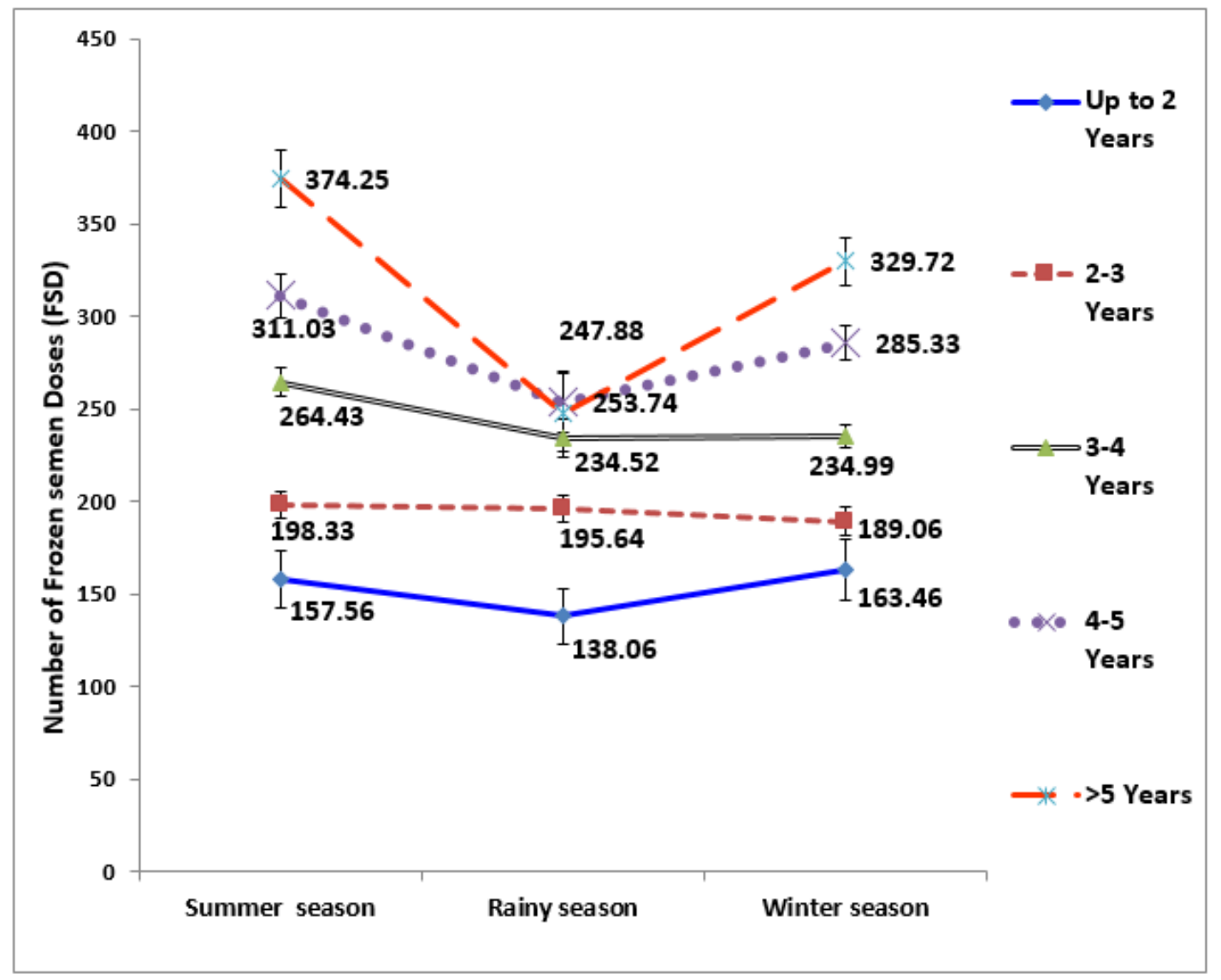

Figure 2

Numbers of frozen semen doses (FSD) production per ejaculate of bulls in different seasons over the age 